\title{
BMJ Open Development of National Cancer Database for Cost and Quality of Life (CaDCQoL) in India: a protocol
}

Shankar Prinja (D) , ${ }^{1}$ Jyoti Dixit, ${ }^{1}$ Nidhi Gupta, ${ }^{2}$ Nikita Mehra, ${ }^{3}$ Ashish Singh, ${ }^{4}$ Manjunath Nookala Krishnamurthy, ${ }^{5}$ Dharna Gupta, ${ }^{1}$ Kavitha Rajsekar, ${ }^{6}$ Jayachandran Perumal Kalaiyarasi, ${ }^{3}$ Partha Sarathi Roy, ${ }^{7}$ Prabhat Singh Malik, ${ }^{8}$ Anisha Mathew, ${ }^{8}$ Awadhesh Pandey, ${ }^{9}$ Pankaj Malhotra, ${ }^{10}$ Sudeep Gupta (D) ,11 Lalit Kumar, ${ }^{8}$ Amal Kataki, ${ }^{12}$ Gurpreet Singh ${ }^{13}$

\section{ABSTRACT}

Introduction The rising economic burden of cancer on healthcare system and patients in India has led to the increased demand for evidence in order to inform policy decisions such as drug price regulation, setting reimbursement package rates under publicly financed health insurance schemes and prioritising available resources to maximise value of investments in health. Economic evaluations are an integral component of this important evidence. Lack of existing evidence on healthcare costs and health-related quality of life (HRQOL) makes conducting economic evaluations a very challenging task. Therefore, it is imperative to develop a national database for health expenditure and HRQOL for cancer.

Methods and analysis The present study proposes to develop a National Cancer Database for Cost and Quality of Life (CaDCQoL) in India. The healthcare costs will be estimated using a patient perspective. A cross-sectional study will be conducted to assess the direct out-of-pocket expenditure (0OPE), indirect cost and HRQOL among cancer patients who will be recruited at seven leading cancer centres from six states in India. Mean 00PE and HRQOL scores will be estimated by cancer site, stage of disease and type of treatment. Economic impact of cancer care on household financial risk protection will be assessed by estimating prevalence of catastrophic health expenditures and impoverishment. The national database would serve as a unique open access data repository to derive estimates of cancer-related OOPE and HRQOL. These estimates would be useful in conducting future cost-effectiveness analyses of management strategies for value-based cancer care.

Ethics and dissemination Approval was granted by Institutional Ethics Committee vide letter no. PGI/IEC03/2020-1565 of Post Graduate Institute of Medical Education and Research, Chandigarh, India. The study results will be published in peer-reviewed journals and presented to the policymakers at national level.

\section{INTRODUCTION}

Traditionally, the burden of cancer is measured in terms of the health outcomes like mortality and morbidity. However, economic
Strengths and limitations of this study

- This study would lead to development of the first national database for costs and quality of life among patients with cancer in India.

- The patient costs and health-related quality of life scores will be determined by cancer site, stage of disease and type of treatment.

- The economic impact of cancer care on household financial risk protection will be assessed.

- Being a hospital-based study, we will not capture quality of life of cancer patients who do not seek care.

- The health system costs on cancer care will not captured in this study, as these are available in National health system cost database.

measures are equally important for cancer outcome research. ${ }^{1}$ These include the cost of services or lost wages incurred as a result of the disease and its treatment. ${ }^{2}$ The cost of cancer has gained considerable importance internationally, given the rising healthcare costs and its financial consequences. High out-of-pocket payments and the indirect costs associated with cancer treatment, often result in financial toxicity. ${ }^{3-5}$ Therefore, characterisation and prediction of these costs, alongside other health outcomes such as both quantity and quality of life, is important for planning strategies to mitigate the financial hardship.

Second, considering the increasing costs of diagnostics and therapeutic interventions for cancer, their formal assessment is imperative to inform value-based standard treatment guidelines. ${ }^{6}$ Economic evaluations are increasingly used to inform the allocation of healthcare funds to ensure best value for money being spent. In order to facilitate such analyses in providing the evidence for priority setting, strong information systems will need 
to be put in place. ${ }^{7}$ India has established a health technology assessment agency (HTAIn), which commissions economic evaluation of new interventions, drugs, diagnostics and treatment strategies. The draft Indian reference case for undertaking economic evaluation as part of health technology assessment (HTA), recommends the use of quality adjusted life years (QALYs) as an index to measure the health consequences. ${ }^{8}$ Computing QALYs requires valuation of health related quality-oflife (HRQOL) or utility scores for different health states. Estimating the utility scores by collecting primary data in each study is time consuming and resource intensive. A database of HRQOL scores for different health states of cancer patients would go a long way to facilitate quick HTA analyses.

The second important evidence need for HTA analyses is cost data. In the context of health financing in India, cost of a service comprises of two parts-health system cost and out-of-pocket expenditure (OOPE). A national health system cost database has been recently created. ${ }^{10}$ Another nationally representative study to measure health system cost of tertiary care hospitals, which includes oncology services, is being carried out in more than 100 hospitals in 11 states. ${ }^{11}$ For OOPE, while National Sample Surveys assess the expenditure for all types of morbidities, the sample of patients with cancer in this data is a mere 500 at all-India level. Several types of specific cancers do not even have a single case. ${ }^{12}{ }^{13}$ Hence the main gap in evidence for conducting HTA is robust data for OOPE among cancer patients, which can be stratified by type of cancer, its health states, levels of severity and type of treatment.

The HTAIn in India has recently commissioned a study to evaluate the value-based prices for 42 anticancer drugs, which have come under price regulation. ${ }^{14}$ Several cancer treatments will be evaluated on grounds of costeffectiveness. As part of this study, primary data will be collected from cancer patients on costs and HRQOL, which will help to develop a national database of patient costs and quality of life among patients with cancer in India (CaDCQoL). This database would serve to build an open-access data repository to derive estimates of cancerrelated medical care costs borne by the patients, indirect costs due to loss of productivity and HRQOL by type of cancer, stage or severity, as well as by treatment approach. This protocol paper provides the detailed description of data collection plan to be followed for assessment of OOPE and HRQOL for each specific type of cancer by stage, site and treatment approach.

\section{METHODS AND ANALYSIS}

The healthcare costs will be estimated using the patient's perspective. A cross-sectional study will be conducted to recruit cancer patients at purposively selected seven public healthcare facilities providing cancer care in India.

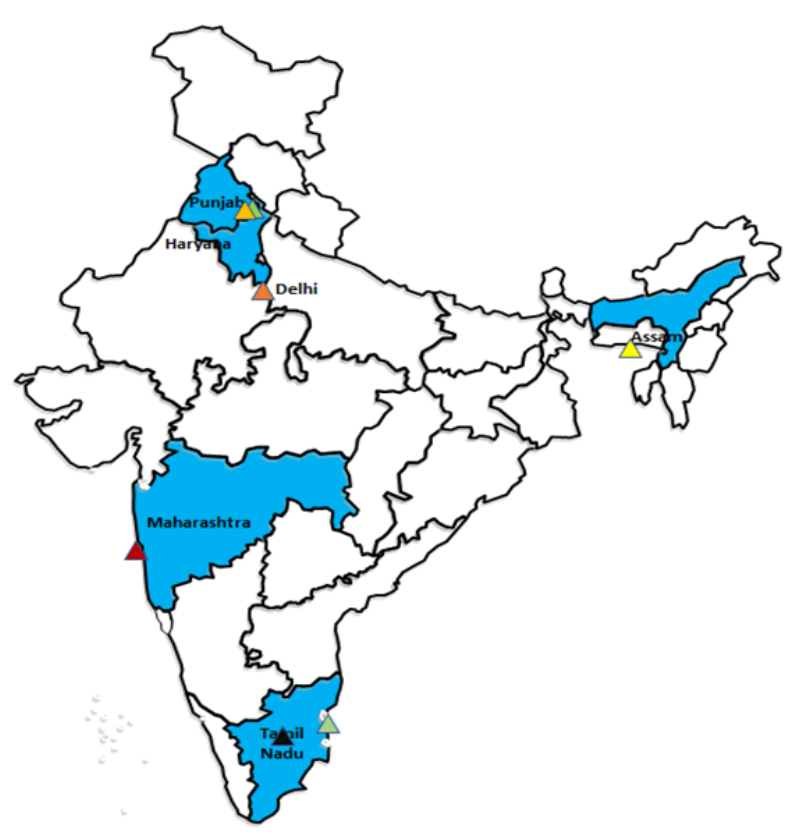

Figure 1 Selected states for proposed study.Each triangle represents a study hospital.

\section{Selection of healthcare facilities}

A multistage stratified sampling technique was followed to recruit cancer patients. In the first stage, the states/ regions were selected on the basis of epidemiological transition level (ETL) of top 10 cancers in India. The ETL state groups were defined on the basis of the trends of top 10 cancer types responsible for the highest proportion of cancer disability-adjusted life years (DALYs) in India. ${ }^{15}$ The states with a relatively lower ratio of DALYs from communicable, maternal, neonatal and nutritional diseases to those from non-communicable diseases including cancer and injuries combined in 2016 indicate higher ETL. Therefore, the ETL state groups were classified as low level ETL state group (ratio 0.56-0.75), middle ETL state group (0.31-0.55) and high ETL state group (less than 0.31). Among high ETL states, Chandigarh (Punjab) and Tamil Nadu were randomly selected. Similarly among middle and low ETL states, Delhi and Maharashtra and Assam were selected respectively. The selection of these states also ensures geographical representation of the country as shown in figure 1 . Inclusion of Assam ensures presence of northern-east region, which has been reported to have differences in patterns of cancer, owing to significant difference in risk factors. ${ }^{16}$ At the second stage, seven healthcare facilities were purposively selected in order to choose hospitals in these states which cater to largest volume of oncology patients. Two of the selected hospitals in our sample, are among the top 10 hospitals in terms of cancer treatment claims as part of the largest insurance scheme in India-the Ayushman Bharat Pradhan Mantri Jan Arogya Yojana (ABPM-JAY). ${ }^{17}$ At the third stage, probability proportional to size (PPS) method will be used to select patients from each of the 


\section{Patient recruitment at outpatient department}
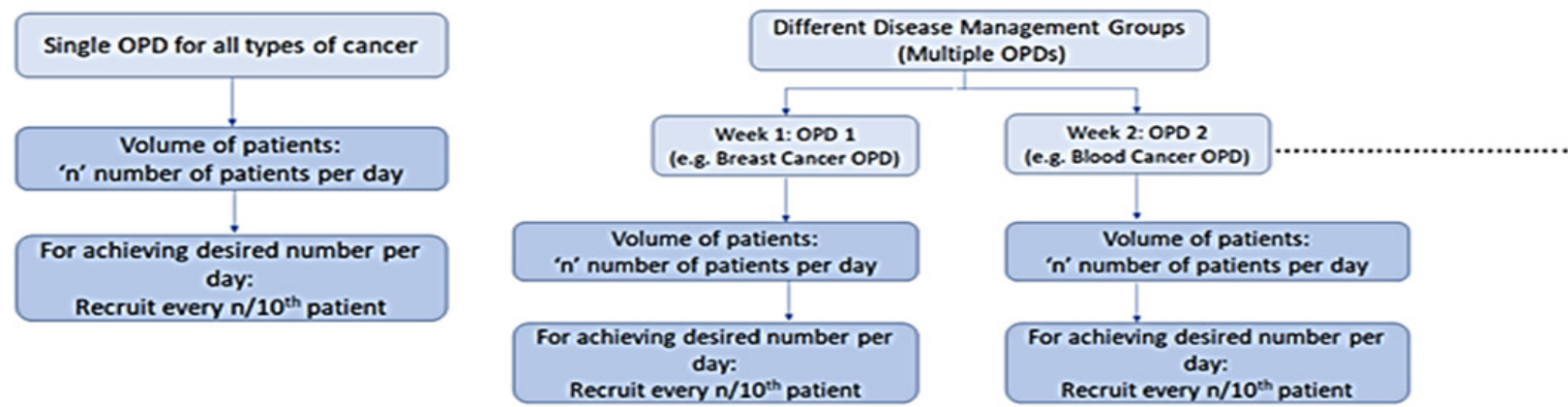

Patient recruitment at inpatient department

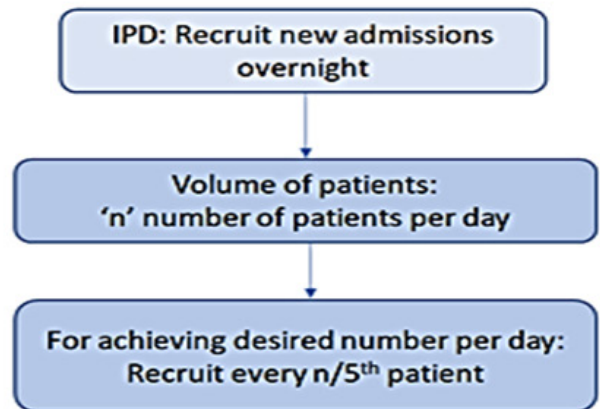

Figure 2 Sampling strategy for patient recruitment. OPD, outpatient department; IPD, inpatient department.

disease management groups (DMGs) in these selected healthcare facilities.

\section{Patient recruitment}

Sampling technique

The patients will be recruited prospectively at outpatient and inpatient departments of the selected public healthcare facilities. The period of data collection at each centre will be 14 months. However, since the start of data collection may not be same across all centres, the data collection will spread out over October 2020 to December 2021. A systematic random sampling technique will be used to recruit the patients using sampling interval chosen on the basis of an average daily number of patients in each health facility, and to achieve the desired sample size. This is applicable to healthcare facilities having common clinics for treating all types of cancers. However, in case of stand-alone cancer centres with different clinics representing different DMGs, a sample of patients would be recruited from each of the DMGs using the PPS method. An illustration of the detailed sampling strategy is shown in figure 2.

\section{Sample size}

Considering the mean OOPE of ₹57 232 with SD of ₹86 871 at $95 \%$ CI and 5\% margin of error, a sample size of 1536 was estimated for each of the seven participating centres. ${ }^{18}$ Taking non-response rate of $10 \%$, the minimum sample size at each centre is estimated as 1690 . We also estimated sample size requirements for other two primary endpoints of the study-catastrophic health expenditures (CHEs) and HRQOL. Since the prevalence of CHE among cancer patients in India has been reported to range from $28 \%$ to $79 \%,{ }^{13} 1819$ we used the mean prevalence of CHE as 50\% at 5\% margin of error and 95\% CI. Based on these assumptions, a sample of 768 was found suitable to measure CHE. Taking a non-response rate of $10 \%$, the final sample size at each centre was estimated as 845 . Similarly, considering the mean utility score of 0.61 among cancer patients in India as reported by Ramasubbu et $a l^{20}$, the minimum sample size for HRQOL assessment was calculated as 362 at $5 \%$ margin of error and 95\% CI. Taking non-response rate of $10 \%$, final sample size for each centre was found to be 398. As the OOPE estimation yields the highest sample size, the minimum of 1690 patients will be interviewed for assessment of OOPE, HRQOL and CHE at each participating centre. With a total of 7 collaborating centres, the study will recruit 11 832 patients with cancer.

\section{Inclusion criteria}

The patients diagnosed with any type of cancer irrespective of age and gender seeking hospitalised and nonhospitalised treatment for any stage at selected healthcare facilities will be included in the study. The study will recruit three types of cancer patients in outpatient setting viz. newly diagnosed (who have been recently diagnosed with cancer), on-treatment (patients who are on some form of active cancer treatment like chemotherapy/ 
radiotherapy, etc) and follow-up cases (patients whose treatment has been completed and are on maintenance therapy). The inpatient department includes cancer wards, high-dependency unit (HDU), intensive care unit (ICU) etc. Newly admitted cancer patients who have been hospitalised overnight (last 24 hours) due to cancer will be recruited. Each patient will be followed up on a daily basis till discharge for capturing information on expenses incurred during last 24 hours. The HRQOL will be assessed on the day of the recruitment. The case definitions used for patient recruitment at outpatient and inpatient settings are described in online supplemental appendix S1.

\section{Data collection instruments}

The outpatients and hospitalised patients will be interviewed using pretested structured interview schedule to collect information on sociodemographic characteristics, household consumption expenditure, clinical data, OOPE and quality of life (see online supplemental appendices S2 and S3). Data on indirect costs will also be elicited by interviewing both patients and their caregivers. Indirect costs refer to the value of time lost because the patient and the caregivers are unable to carry out normal productive activities because of cancer. Therefore, patients and all their caregivers (one or multiple) accompanying the patient while seeking non-hospitalised treatment as well as during the period of hospitalisation, will be interviewed to estimate indirect costs using a pretested structured interview schedule given in online supplemental appendix S4. During patient interviews at outpatient setting, the newly diagnosed, on-treatment patients and follow-up cases who sought care within the last 30 days will be interviewed for the direct medical expenditure incurred since the last visit for non-hospitalised treatment such as outpatient consultation, diagnostic tests, day-care sessions (chemotherapy or radiotherapy), drugs purchased from pharmacy stores etc. along with the indirect costs due to loss of productivity. Thus, the mean OOPE since the last hospital visit or during the past 30 days since the present visit, whichever is less (if the last visit was less than 30 days ago) would be estimated.

Additionally, patients recruited in outpatient setting will also be interviewed for any episode of hospitalisation, type of hospital (whether public/private), expenditure incurred on hospitalisation during last 1 year and the source of financing healthcare expenditure. However, follow-up cases who sought care more than 30 days ago will be interviewed telephonically at 15 th day following recruitment to elicit data on expenditure incurred on treatment since their last visit to estimate per visit OOPE on cancer. Other information such as socio-demographic characteristics, morbidity profile, consumption expenditure, OOPE and HRQOL will be captured on the day of recruitment. This is because such follow-up cases with last visit more than 30 days ago are less likely to recall the expenditure incurred since the last visit. ${ }^{21}$ At the time of recruitment, the investigators will be trained to develop a good rapport with patients, and a minimum of 2-3 contact numbers will be elicited to ensure high response rate on day 15th over telephone. Further, patients with cancer require repetitive contact with healthcare providers owing to the nature of the disease and hence it is likely that patients with cancer will be more responsive during follow-up. Such a methodology of collecting information on OOPE using telephonic interviews has been reported to be valid in several previous studies reporting the follow-up rate of more than $80 \%$ using telephonic interviews. ${ }^{22} 23$

The generic HRQOL data will be collected using EuroQol 5-Dimensional 5-Level (EQ-5D-5L) for estimation of utility scores among patients of different cancers. $^{24}$ EQ-5D-5L is a generic questionnaire intending to cover five attributes of well-being: mobility, self-care, usual activity, pain/discomfort and anxiety/ depression. ${ }^{24}{ }^{26}$ Each attribute of EQ-5D-5L has five levels: (1) no problems, (2) slight problems, (3) moderate problems, (4) severe problems and (5) extreme problem. The summary of data collection plan for outpatients is shown is figure 3 .

Similarly, patients who have been hospitalised overnight will be interviewed daily till discharge to collect information on direct medical expenses incurred during last 24 hours on hospitalisation including inpatient stay in cancer ward/HDU/ICU/surgical procedure in inpatient setting etc., indirect costs due to loss of wages as well as HRQOL. However, rest of the information such as socio-demographic characteristics, clinical information, consumption expenditure will be recorded on the day of recruitment. The bills of expenditure incurred on medicines, hospital charges, procedure, diagnostics etc. will be obtained to improve the accuracy of data.

\section{Data analysis}

The data on quality of life will be analysed to compute utility scores using Indian tariff value set. ${ }^{27}$ This would be the first study in India on valuation of health outcomes to use the Indian tariff values to determine utility weights for patients with cancer. The mean OOPE along with SE will be computed with respect to type of cancer, health state, type of treatment (chemotherapy, radiotherapy, surgery, etc) and setting (outpatient, hospitalisation, intensive care) using clinical information obtained. For calculating indirect costs, human capital approach will be used. ${ }^{28}$ Loss of working days will be recorded, that is the days that a person (patient as well as the caregiver) missed or remained absent from his/her work due to hospital stay. For those actively employed in the labour workforce, per day income as reported by the individual will be used for calculations. For individuals not part of the workforce, an average minimum daily wage rate for India specific to gender and area of residence (rural/urban) will be imputed. ${ }^{29}$ The extent of financial risk protection (FRP) will be assessed in terms of CHE, impoverishment and distress financing. Expenditure on cancer treatment which exceeds the threshold of $40 \%$ of non-food 


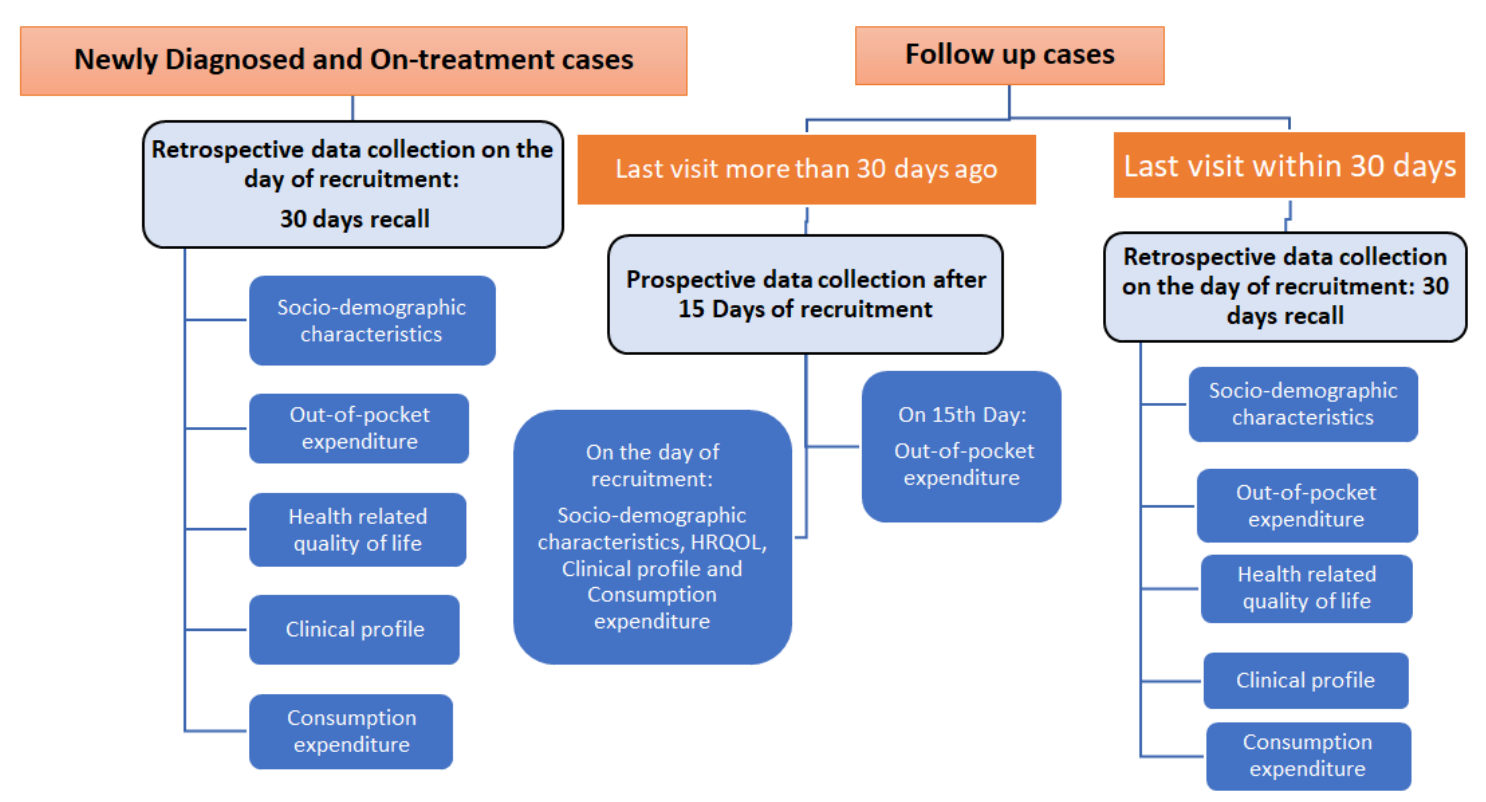

Figure 3 Data collection plan for different types of cancer patients. HRQOL, health-related quality of life.

household consumption expenditure will be considered as CHE. ${ }^{30}$ Households which have either borrowed money (with or without interest) or have sold their assets (like land, home, cattle, etc) to cope with the expenditure will be classified to have faced distress financing. ${ }^{32} 33$

Multiple logistic regression analysis will be performed to examine the association of CHE and the distress financing with factors including age, sex, income status, treatment modality, insurance status, locality and stage at the time of diagnosis. A sensitivity analysis will be carried out to assess the prevalence of catastrophic expenditure at varying cut-off levels, that is, $20 \%-50 \%$. Impoverishment will be calculated in terms of relative increase in poverty headcount. We will compare poverty head counts before and after OOP payments for hospitalisation. The prehospitalisation poverty headcount (Pre Hp) will be calculated using mean per-capita consumption expenditure (xi) using the Tendulkar committee's poverty line (PL) cut-off of INR 961 per person per month. ${ }^{34}$

Pre $\mathrm{Hp}=1 / \mathrm{n} \sum(\mathrm{xi} \leq \mathrm{PL})$

Where $\mathrm{n}=$ number of individual

The posthospitalisation poverty headcount will also be computed in a similar manner by netting out OOP payments for hospitalisation from consumption expenditure and then comparing with poverty line

Post $\mathrm{Hp}=1 / \mathrm{n} \sum(\mathrm{xi}-\mathrm{OOP} \leq \mathrm{PL})$

Where $\mathrm{n}=$ number of individuals.

\section{Study outcomes}

- Mean per visit OOPE incurred on non-hospitalised treatment of cancer.

- Mean OOPE incurred per episode of hospitalisation stratified by ward, ICU and HDU.

- Mean OOPE incurred per surgical intervention.
- Mean OOPE for outpatients and inpatients, by type of treatment, disease severity, level of healthcare utilisation, type of healthcare provider, etc.

- CHE, impoverishment and distress financing due to cancer treatment.

- HRQOL of patients with cancer, by type of cancer, treatment, disease stage, complications, etc.

\section{Patient and public involvement}

Patients will be involved in the present study to capture information on direct medical expenditure incurred on cancer treatment, indirect costs due to loss of productivity and HRQOL. A written informed consent will be obtained from all study participants who are more than 18 years of age. In case of severely ill or critical patients, we would wait for the patient's condition to improve and then interview the patient to collect the required information. This is important since the evidence suggests a need to preferably interview the person affected to elicit data on HRQOL. ${ }^{35}$ Accordingly, we will obtain informed consent also from the severely ill patient.

However, in case of minors, the informed consent will be obtained from parents/guardians. Further, in case of minors information on OOPE and HRQOL will be gathered from parents/guardians and proxy respondents (care providers) respectively. This has also been done in several previous studies. ${ }^{36} 37$

\section{DISCUSSION}

Universal Health Coverage (UHC) is a long-standing tenet of global health and has, in recent years become the overarching framework for policies and investments in health globally and nationally. ${ }^{38}$ FRP is a key component of UHC, which is defined as access to all needed 
quality health services without financial hardship. ${ }^{39}$ In order to realise UHC, cancer services must be included in benefit packages and sustainably financed through public resources for protecting the cancer patients against financial toxicity. Financial toxicity has been shown to affect access to cancer care, leading to delay or foregoing cancer care, bankruptcy, poor quality of life and poor survival. ${ }^{40}{ }^{41}$ Therefore, it is important to acknowledge financial toxicity as an important outcome of clinical condition impacting patients with cancer, as well as to identify the most actionable and effective interventions to prevent financial hardship, in order to deliver UHC by United Nations. ${ }^{42}$

Once the governments commit to the aspirational goal of financing health services to provide universal coverage, healthcare systems face the challenge of fiscal sustainability in the context of scarce resources. This becomes even more evident in the context of oncology due to high cost of care. As a result, decisions regarding priority setting become inevitable. It is thus no surprise that two out of the initial thirteen studies commissioned by HTAIn are focused on evaluating strategies for cancer screening and prevention in India. ${ }^{43}$ Moreover, a large multicentric study is being carried out to determine the value-based pricing guidelines for anticancer drugs. ${ }^{14}$ The present study protocol part is of this larger study.

It is also worthwhile to mention that National Cancer Grid in collaboration with National Health Agency, India have signed a memorandum of Understanding to strengthen delivery of cancer services under ABPM-JAY with common objectives to reduce cancer burden, ensure uniform standards of patient care towards effective and efficient patient-centric care, improve access to cancer services and ensure FRP. ${ }^{445}$ These agencies also support the use of HTA in informing policy decisions and thereby achieving the sustainable development goals on the pathway to attain UHC. However, the lack of cost data represents a major evidence barrier in the journey towards UHC-oriented health policy decisions in India. In particular, in setting reimbursement package rates, the limited availability of cost information is seen as a significant concern. ${ }^{114647}$

Several countries have established such databases of healthcare costs. ${ }^{48}$ India has also created a national database of health system costs. ${ }^{10} 46$ However, none of these national databases have a specific focus on cancer. Moreover, due to being a generic database of cost, it does not provide disaggregated data on cost by the type of disease and level of severity. Finally, the existing database contains only estimates of health system cost, while nearly $68 \%$ of the total health expenditure in India is financed out-of-pocket by households. As a result, the proposed CaDCQoL study would be a significant value addition. Since the study aims to determine the patient care costs which will help in determining provider payment rates, conduct cost-effectiveness analyses for value based care, the study has chosen to include public hospitals. The patient care costs derived from data collected from these public hospitals can be aggregated with health system costs estimated in another national costing study conducted in public hospitals to compute the overall societal cost of cancer care in India. ${ }^{46}$ Although, the present study has no private hospital as such but three hospitals in our sample have category of patients who are provided care at prices similar to the prices of the most pure private hospitals in tier 2 and 3 cities of India. The study will provide comprehensive estimate of economic burden attributable to cancer in India. Using the distribution of cancers as per National Cancer Registry Programme, ${ }^{49}$ and the consequent expected number of individual cancer patients likely to be recruited with a total sample of 11832 patients, we estimate that our study sample would be sufficient to provide valid estimates of OOPE, CHE and HRQOL for top 3, 6 and 12 cancers, respectively, in India, with a 5\% margin of error and $95 \%$ CI. However, at $10 \%$ margin of error and $95 \%$ CI, our study would be powered to give valid estimates of OOPE, CHE and HRQOL for top 11, 17 and 20 cancers, respectively, in India as shown in table 1.

Further, several countries have published their valuesets for different health states using the EQ-5D-5L. ${ }^{50-52}$ The HTAIn in India has also recently completed a study and will shortly publish its own value set. ${ }^{27}$ However, it is not possible to cross-walk the health state with the individual cancer patients and their stage and type of treatment. On the other hand, the HTA study precisely requires information on the latter. As a result the present study would add significantly to the existing evidence base by providing stage-specific, and severity-specific estimates of utility score for cancer patients.

\section{Future applications of database of healthcare costs and HRQOL}

The national database developed as part of this study will serve as unique Indian data repository of cost of cancer care as well as HRQOL. This database would be the sole evidence-based resource on OOPE estimates (both

Table 1 Estimated sample size for OOPE, HRQOL and CHE at 5\% and 10\% margin of error

\begin{tabular}{|c|c|c|c|c|c|c|}
\hline \multirow[b]{2}{*}{ Margin of error } & \multicolumn{3}{|c|}{ Sample size } & \multicolumn{3}{|c|}{ No of cancers with valid estimates } \\
\hline & OOPE & CHE & HRQOL & OOPE & CHE & HRQOL \\
\hline $5 \%$ & 1690 & 845 & 398 & 3 & 6 & 12 \\
\hline $10 \%$ & 422 & 211 & 99 & 11 & 17 & 20 \\
\hline
\end{tabular}

$\mathrm{CHE}$, catastrophic healthcare expenditure; HRQOL, health-related quality of life; OOPE, out-of-pocket expenditure. 
direct and indirect costs), which along with the health system costs, can be used to inform the provider payment rates for cancer specific health benefit packages under various national and state-level publicly financed health insurance schemes. Further, the estimated OOPE and HRQOL by site, stage and treatment approach will aid in robust cost-effectiveness analysis of screening and treatment strategies for cancer control in India. Currently, there are few published and readily accessible cost data in India particularly on cancer to inform HTA and insurance design. The present study is going to be a first step in providing easily accessible reference cost and HRQOL data on cancer for India. The study findings will be available through the National health system cost database besides publishing as peer-reviewed papers. ${ }^{10}$

\section{Author affiliations}

${ }^{1}$ Department of Community Medicine and School of Public Health, Post Graduate Institute of Medical Education and Research, Chandigarh, Chandigarh, India ${ }^{2}$ Department of Radiation Oncology, Government Medical College and Hospital, Chandigarh, Chandigarh, India

${ }^{3}$ Department of Medical Oncology, Cancer Institute-WIA, Chennai, Tamil Nadu, India ${ }^{4}$ Department of Medical Oncology, Christian Medical College Vellore, Vellore, Tamil Nadu, India

${ }^{5}$ Department of Clinical Pharmacology, Tata Memorial Centre, Mumbai, Maharashtra, India

${ }^{6}$ Department of Health Research, India Ministry of Health and Family Welfare, New Delhi, Delhi, India

${ }^{7}$ Department of Medical Oncology, Dr B Borooah Cancer Society Trust, Guwahati, Assam, India

${ }^{8}$ Department of Medical Oncology, AllMS, New Delhi, Delhi, India

${ }^{9}$ Radiotherapy and Oncology, Government Medical College and Hospital, Chandigarh, Chandigarh, India

${ }^{10}$ Department of Internal Medicine, Post Graduate Institute of Medical Education and Research, Chandigarh, Chandigarh, India

${ }^{11}$ Medical Oncology, Tata Memorial Hospital, Mumbai, Maharashtra, India

${ }^{12}$ Department of Gynaecologic Oncology, Dr B Borooah Cancer Society Trust, Guwahati, Assam, India

${ }^{13}$ Department of General Surgery, Post Graduate Institute of Medical Education and Research, Chandigarh, Chandigarh, India

Contributors Study conception: SP, LK, SG, AK and PM. Study design: SP, NG, JD and DG. Study tools: SP, NG, JD, DG, NM, AS, MNK, KR, JPK, PSR, PSM, AM, AP, PM, SG, LK, AK and GS. Writing (first draft): JD and SP. Writing (review and editing): SP, JD, NG, NM, AS, MNK, DG, KR, JPK, PSR, PSM, AM, AP, PM, SG, LK, AK and GS.

Funding The study is funded by the Department of Health Research, Ministry of Health and Family Welfare, Government of India vide grant number F.No.T.11011/02 /2017-HR/3100291.

Map disclaimer The depiction of boundaries on this map does not imply the expression of any opinion whatsoever on the part of BMJ (or any member of its group) concerning the legal status of any country, territory, jurisdiction or area or of its authorities. This map is provided without any warranty of any kind, either express or implied.

Competing interests None declared.

Patient consent for publication Not required.

Provenance and peer review Not commissioned; externally peer reviewed.

Supplemental material This content has been supplied by the author(s). It has not been vetted by BMJ Publishing Group Limited (BMJ) and may not have been peer-reviewed. Any opinions or recommendations discussed are solely those of the author(s) and are not endorsed by BMJ. BMJ disclaims all liability and responsibility arising from any reliance placed on the content. Where the content includes any translated material, BMJ does not warrant the accuracy and reliability of the translations (including but not limited to local regulations, clinical guidelines, terminology, drug names and drug dosages), and is not responsible for any error and/or omissions arising from translation and adaptation or otherwise.

Open access This is an open access article distributed in accordance with the Creative Commons Attribution Non Commercial (CC BY-NC 4.0) license, which permits others to distribute, remix, adapt, build upon this work non-commercially, and license their derivative works on different terms, provided the original work is properly cited, appropriate credit is given, any changes made indicated, and the use is non-commercial. See: http://creativecommons.org/licenses/by-nc/4.0/.

ORCID iDs

Shankar Prinja http://orcid.org/0000-0001-7719-6986

Sudeep Gupta http://orcid.org/0000-0002-6742-6378

\section{REFERENCES}

1 Sharma K, Das S, Mukhopadhyay A, et al. Economic cost analysis in cancer management and its relevance today. Indian $\mathrm{J}$ Cancer 2009;46:184-9.

2 Fryback DG, Craig BM. Measuring economic outcomes of cancer. J Natl Cancer Inst Monogr 2004;33:134-41.

3 Lentz R, Benson AB, Kircher S. Financial toxicity in cancer care: prevalence, causes, consequences, and reduction strategies. J Surg Oncol 2019;120:85-92 https://doi.org/10.1002/jso.25374

4 Mehlis K, Witte J, Surmann B, et al. The patient-level effect of the cost of cancer care - financial burden in German cancer patients. BMC Cancer 2020;20:529

5 Carrera PM, Kantarjian HM, Blinder VS. The financial burden and distress of patients with cancer: understanding and stepping-up action on the financial toxicity of cancer treatment. CA Cancer J Clin 2018:68:153-65.

6 Smith PC. Measuring for value for money in health care: concepts and tools, 2009. Available: http://www.health.org.uk/sites/health/ files/MeasuringValueForMoneyInHealthcareConceptsAndTools.pdf [Accessed 03 Aug 2016].

7 Downey L, Rao N, Guinness L, et al. Identification of publicly available data sources to inform the conduct of health technology assessment in India. F1000Res 2018;7:245.

8 Downey L, Rao N, Guinness L, et al. Identification of publicly available data sources to inform the conduct of health technology assessment in India. F1000Res 2018;7:245.

9 Rajsekar K. Indian reference case for undertaking economic evaluation for health technology assessment in India. New Delhi: Department of Health Research, Ministry of Health and Family Welfare, Government of India, 2018.

10 Prinja S, Selvaraj S, Muraleedharan V. National health system cost database for India, 2019. Available: https://www.healtheconomics. pgisph.in/costing_web/index.php?action=gen_secondary [Accessed 24 Aug 2019].

11 Prinja S, Singh MP, Guinness L, et al. Establishing reference costs for the health benefit packages under universal health coverage in India: cost of health services in India (CHSI) protocol. BMJ Open 2020;10:e035170.

12 National Sample Survey Office. Key indicators of social consumption in India- health, NSs 71st round (January-June 2014. New Delhi: National Sample Survey Office, Ministry of Statistics and Programme Implementation, Government of India, 2015.

13 Rajpal S, Kumar A, Joe W. Economic burden of cancer in India: evidence from cross-sectional nationally representative household survey, 2014. PLoS One 2018;13:e0193320.

14 HTA study proposals and outcome report, 2020. Available: https:// htain.icmr.org.in/images/pdf/16th_TAC_Meeting_Minutes.pdf [Accessed 15 Dec 2020].

15 India State-Level Disease Burden Initiative Cancer Collaborators. The burden of cancers and their variations across the states of India: the global burden of disease study 1990-2016. Lancet Oncol 2018;19:1289-306.

16 Mathur P, Sathishkumar K, Chaturvedi M, et al. Cancer statistics, 2020: report from National cancer registry programme, India. JCO Glob Oncol 2020;6:1063-75.

17 Kaur S, Jain N, Bhatnagar P. Early Trends From Utilization Of Oncology Services: Insights From Ayushman Bharat Pradhan Mantri Jan Arogya Yojana (PMJAY). [online], 2020. Available: <https://pmjay. gov.in/sites/default/files/2019-11/Working\%20paper-4\%20(1).pdf> [Accessed 24 Dec 2020].

18 Kastor A, Mohanty SK. Disease-specific out-of-pocket and catastrophic health expenditure on hospitalization in India: do Indian households face distress health financing? PLOS One 2018;13:1-18. 
19 Singh MP, Chauhan AS, Rai B, et al. Cost of treatment for cervical cancer in India. Asian Pac J Cancer Prev 2020;21:2639-46.

20 Ramasubbu SK, Pasricha RK, Nath UK, et al. Quality of life and factors affecting it in adult cancer patients undergoing cancer chemotherapy in a tertiary care hospital. Cancer Rep 2021;4:e1312.

21 Prinja S, Jagnoor J, Chauhan AS, et al. Economic burden of hospitalization due to injuries in north India: a cohort study. Int $J$ Environ Res Public Health 2016;13:pii: E673.

22 Prinja S, Jagnoor J, Sharma D. Out-Of-Pocket expenditure and financial risk protection for hospitalization due to injuries in public sector hospitals in North India. PLoS One 2019;14:e0224721.

23 Jagnoor J, Prinja S, Nguyen H, et al. Mortality and healthrelated quality of life following injuries and associated factors: a cohort study in Chandigarh, North India. Inj Prev 2020;26:pii: injuryprev-2019-043143.

24 The EuroQol Group. EQ-5D is a recommended tool for use in costutility analyses around the globe [Internet], 2018. Available: https:// euroqol.org/eq-5d-is-a-recommended-tool-for-use-in-cost-utilityanalyses-around-the-globe/ [Accessed 24 Dec 2018].

25 Rabin R, de Charro F. EQ-5D: a measure of health status from the EuroQol group. Ann Med 2001;33:337-43.

26 Brooks R. EuroQol: the current state of play. Health Policy 1996;37:53-72.

27 Jyani G, Prinja S, Kar SS, et al. Valuing health-related quality of life among the Indian population: a protocol for the development of an $E Q-5 D$ value set for India using an extended design (DEVINE) study. BMJ Open 2020;10:e039517.

28 Rice DP, Hodgson TA, Kopstein AN. The economic costs of illness: a replication and update. Health Care Financ Rev 1985;7:61-8.

29 Papola TS, Kanan KP. ILO-Asia Pacific working paper series. towards an India wage report,DWT for South Asia and Country Office for India; 2017: 185p.

$30 \mathrm{Xu}$ K. Distribution of health payments and catastrophic expenditures methodology. Geneva: World Health Organization, 2005.

31 Moreno-Serra R, Millett C, Smith PC. Towards improved measurement of financial protection in health. PLoS Med 2011:8:e1001087.

32 Huffman MD, Rao KD, Pichon-Riviere A, et al. A cross-sectiona study of the microeconomic impact of cardiovascular disease hospitalization in four low- and middle-income countries. PLoS One 2011;6:e20821.

33 George S, Balachandrab A. The economic burden of anticancer medicines for households in India: evidence from data on expenditure on medicine and drug prices, 2019. Available: https:// papers.ssrn.com/sol3/DisplayAbstractSearch.cfm

34 GAUR S, RAO N. Poverty measurement in india: A status update [Internet], 2020. Available: https://rural.nic.in/sites/default/files/ WorkingPaper_Poverty_DoRD_Sept 2020.pdf [Accessed 26 Dec 2020].

35 Arafa MA, Zaher SR, El-Dowaty AA, et al. Quality of life among parents of children with heart disease. Health Qual Life Outcomes 2008;6:91.

36 Rensen N, Steur LMH, Schepers SA, et al. Determinants of healthrelated quality of life proxy rating disagreement between caregivers of children with cancer. Qual Life Res 2020;29:901-12.
37 Eiser C, Morse R. Morse RA review of measures of quality of life for children with chronic illness. Arch Dis Child 2001;84:205-11.

38 Addressing cancer control within universal health coverage, 2020. Available: https://www.uicc.org/sites/main/files/atoms/files/UICC Report_Universal-Health\%20Coverage_FA.pdf [Accessed 21 Sep 2020].

39 Saksena P, Hsu J, Evans DB. Financial risk protection and universal health coverage: evidence and measurement challenges. PLoS Med 2014;11:e1001701

40 Perrone F, Jommi C, Di Maio M, et al. The association of financial difficulties with clinical outcomes in cancer patients: secondary analysis of 16 academic prospective clinical trials conducted in Italy. Ann Oncol 2016;27:mdw433.

41 Zafar SY. Financial toxicity of cancer care: it's time to intervene. Natl Cancer Inst 2016;108. doi:10.1093/jnci/djv370. [Epub ahead of print: 1112 2015].

42 United nations. Transforming our world: the 2030 agenda for sustainable development. 16. New York, NY: UN, 2015.

43 Chauhan AS, Prinja S, Srinivasan R, et al. Cost effectiveness of strategies for cervical cancer prevention in India. PLoS One 2020;15:e0238291.

44 National Health Authority inks partnership with National Cancer Grid to strengthen delivery of cancer services under Ayushman Bharat PM-JAY [Internet], 2020. Available: https://www. countryandpolitics.in/2019/05/23/national-health-authority-inkspartnership-with-national-cancer-grid-to-strengthen-delivery-ofcancer-services-under-ayushman-bharat-pm-jay/ [Accessed 21 Sep 2020]

45 Press Information Bureau, Government of India. Ministry of health and family welfare Ayushman Bharat-Pradhan Mantri Jan AarogyaYojana (AB-PMJAY), 2018. http://pib.nic.in/newsite/ PrintRelease. aspx? relid $=183624$

46 Prinja S, Chauhan AS, Rajsekhar K, et al. Addressing the cost data gap for universal healthcare coverage in India: a call to action. Value Health Reg Issues 2020;21:226-9.

47 Bahuguna P, Guinness L, Sharma S, et al. Estimating the unit costs of healthcare service delivery in India: addressing information gaps for price setting and health technology assessment. Appl Health Econ Health Policy 2020;18:699-711.

48 Yothasamut J, Tantivess S, Teerawattananon Y. Using economic evaluation in policy decision-making in Asian countries: mission impossible or mission probable? Value Health 2009;12 Suppl 3:S26-30.

49 Dhillon P, Mathur P, Nandakumar A, et al. The burden of cancers and their variations across the states of India: the global burden of disease study 1990-2016. Lancet Oncol 2018;19:1289-306.

50 Riewpaiboon A. PRM3 standard cost list for economic evaluation in Thailand. Value in Health 2012;15:A645

51 Chevalier J, de Pouvourville G. Valuing EQ-5D using time trade-off in France. Eur J Health Econ 2013;14:57-66 http://www.ncbi.nlm.nih. gov/pubmed/21935715

52 Gerlinger C, Bamber L, Leverkus F, et al. Comparing the EQ-5D-5L utility index based on value sets of different countries: impact on the interpretation of clinical study results. BMC Res Notes 2019;12:18. 